\title{
GENERALIZATIONS OF KOCHEN AND SPECKER'S THEOREM AND THE EFFECTIVENESS OF GLEASON'S THEOREM
}

\author{
EHUD HRUSHOVSKI AND ITAMAR PITOWSKY
}

\begin{abstract}
Kochen and Specker's theorem can be seen as a consequence of Gleason's theorem and logical compactness. Similar compactness arguments lead to stronger results about finite sets of rays in Hilbert space, which we also prove by a direct construction. Finally, we demonstrate that Gleason's theorem itself has a constructive proof, based on a generic, finite, effectively generated set of rays, on which every quantum state can be approximated.
\end{abstract}

\section{Gleason's Theorem and Logical Compactness}

Kochen and Specker's (1967) theorem (KS) puts a severe constraint on possible hidden-variable interpretations of quantum mechanics. Often it is considered an improvement on a similar argument derived from Gleason (1957) theorem (see, for example, Held. 2000). This is true in the sense that KS provide an explicit construction of a finite set of rays on which no two-valued homomorphism exists. However, the fact that there is such a finite set follows from Gleason's theorem using a simple logical compactness argument (Pitowsky 1998, a similar point is made in Bell 1996). The existence of finite sets of rays with other interesting features also follow from the same simple consideration. In Pitowsky (1998) some such consequences, in particular the "logical indeterminacy principle" are pointed out, and some are given a direct constructive proof. In this section we shall formulate the general compactness principle underlying these results and mention some new ones. In the second section there are some explicit constructions of finite sets of rays whose existence was inferred indirectly in the first section; in particular, a simpler proof of the logical indeterminacy principle. In the last section we prove that there is an effective (algorithmic) procedure to construct finite sets of rays which force a uniform approximation to quantum states. In particular, we provide a short demonstration that Gleason's theorem has a constructive proof, a fact previously established by Richman and Bridges (1999).

Let $\mathbb{H}$ be a Hilbert Space of a finite dimension $n \geq 3$ over the complex or real field. A non negative real function $p$ defined on the unit vectors in $\mathbb{H}$ is called $a$ state on $\mathbb{H}$ if the following conditions hold:

1. $p(\alpha x)=p(x)$ for every scalar $\alpha,|\alpha|=1$, and every unit vector $x \in \mathbb{H}$.

2. If $x_{1}, x_{2}, \ldots, x_{n}$ is an orthonormal basis in $\mathbb{H}$ then $\sum_{j=1}^{n} p\left(x_{j}\right)=1$.

Gleason's theorem characterizes all states:

Theorem 1. Given a state $p$, there is an Hermitian, non negative operator $W$ on $\mathbb{H}$, whose trace is unity, such that $p(x)=(x, W x)$ for all unit vectors $x \in \mathbb{H}$, where $($,$) is the inner product.$

Gleason's (1957) original proof of the theorem has three parts: The first is to show that every state $p$ on $\mathbb{R}^{3}$ is continuous. The second part is a proof of the 
theorem in the case of $\mathbb{R}^{3}$, and the third part is a reduction of the general theorem to $\mathbb{R}^{3}$. The theorem is also valid in the infinite dimensional case which we shall not consider.

Let us make more precise what are the formal logical assumptions underlying the proof of Gleason's theorem. For simplicity we shall concentrate on the three dimensional real case which comprises the first two parts of Gleason's proof. All our results are extendable to any real or complex Hilbert space of a finite dimension $n \geq 3$.

Consider the first order formal theory of the real numbers (that is, a first order theory of some standard model $\mathbb{R}$ of the reals). This induces a theory of $\mathbb{R}^{3}$, together with the inner product, and the unit sphere $\mathbb{S}^{2}$. Add to this first order theory a function symbol $p: \mathbb{S}^{2} \rightarrow \mathbb{R}$. Let the Greek letters $\alpha, \beta, \gamma \ldots$ denote variables ranging over the reals and $x, y, z, \ldots$ be variables ranging over $\mathbb{S}^{2}$. Now add the axioms:

G1. $\forall x p(x) \geq 0$

G2. $\forall x p(-x)=p(x)$.

G3. For each orthonormal triple $x, y, z \in \mathbb{S}^{2}$ an axiom: $p(x)+p(y)+p(z)=1$

Note that in G3 we do not use the universal quantifier. Instead G3 is an axiom schema with a continuum of propositions. The next axiom is just the statement that every set of reals which is bounded from below has an infimum. However, since we want to use only first order formulae we write it as an axiom schema:

G4. For every one place predicate of reals $A($.$) expressible in our language an$ axiom

$\exists \beta \forall \alpha(A(\alpha) \rightarrow \alpha \geq \beta) \longrightarrow \exists \beta[\forall \alpha(A(\alpha) \rightarrow \alpha \geq \beta) \wedge \forall \varepsilon>0 \exists \gamma(A(\gamma) \wedge \beta>\gamma-\varepsilon)]$

Thus, for example, the claim that $p$ itself has an infimum will follow from $\mathbf{G 1}$ and the application of $\mathbf{G} 4$ to the predicate $\exists x(p(x)=\alpha)$. As a matter of fact, the proof of Gleason's theorem requires twice the application of $\mathbf{G 4}$, that is, for two predicates $A($.$) .$

The proof that $p$ is continuous depends on the axioms G1-G4. One can see this from Gleason's (1957) original proof, or more directly from Pitowsky (1998) that only a finite number of application of the schema $\mathbf{G 3}$ are required.

To prove the second part, that every state on $\mathbb{R}^{3}$ is given by a self adjoint, non-negative, trace one operator, another axiom is needed:

G5.If $p$ is continuous then its minimum and maximum are obtained:

$\forall \varepsilon>0 \exists \delta>0 \forall x, y(\|x-y\|<\delta \rightarrow|p(x)-p(y)|<\varepsilon) \longrightarrow \exists x, y \forall z(p(x) \leq p(z) \leq$ $p(y))$.

An elementary way to complete the proof of Gleason's theorem on the basis of G5 is in Cooke et al (1984) or Richman and Bridges (1999). Here the proof is based on a limiting process. Using the continuity of $p$, which has been proved from G1G4, the claim that $p$ obtains its minimum and maximum follows from G5. Using the minimum and maximum points of $p$ one determines the operator $W$, which is the candidate to represent it. Then one proves that for all $\varepsilon>0$ the proposition $\forall x(|p(x)-(x, W x)|<\varepsilon)$ holds, which completes the proof.

With these observations it is easy to see how Kochen and Specker's theorem follows from Gleason's theorem. Consider the proposition:

F1. There is a state $p$ such that $\forall x(p(x)=0 \vee p(x)=1)$

Now, the conjunction of $\mathbf{F 1}$ with $\mathbf{G 1 - G} \mathbf{4}$ is inconsistent, since the latter imply that $p$ is continuous. Hence, there is a proof of a contradiction from $\mathbf{G 1 - G 4 +}$ F1. The proof of that contradiction uses only finitely many cases of the schema 
G3 (since any proof is finite). If one collects the directions $x \in \mathbb{S}^{2}$ which appear in that proof one gets a finite set of directions on which no two valued homomorphism exists. Of course this compactness argument does not yield an explicit set, but it may serve as a incentive to look for one, which might have been Kochen and Specker's motivation. A similar argument was explicitly used by Clifton (1993). He simply lifted the vectors which appear in Bell (1966) simplified 0-1 version of "Gleason's theorem" to obtain a KS theorem. See also Fine and Teller (1978), Pitowsky (1982).

But the argument just presented can be easily generalized to include many more propositions which contradict Gleason's theorem. Let $\Gamma \subset \mathbb{S}^{2}$ be a finite set such that $x \in \Gamma \rightarrow-x \in \Gamma$. We shall say that $p: \Gamma \rightarrow \mathbb{R}$ is a state on $\Gamma$ if $p$ satisfies G1-G3 for all directions in $\Gamma$. Now, consider the statement

F2 There is a state $p$ that has exactly $k$ values $(k \geq 2)$. In other words, $p$ satisfies the proposition:

$\mathcal{A}_{k}=\exists x_{1}, x_{2}, \ldots x_{k} \bigwedge_{i \neq j}\left(p\left(x_{i}\right) \neq p\left(x_{j}\right)\right) \wedge \forall y\left(p(y)=p\left(x_{1}\right)\right) \vee \ldots \vee\left(p(y)=p\left(x_{k}\right)\right)$

This contradicts Gleason's theorem since, again by continuity, if $p$ has two or more values it has infinitely many. Hence, for all $k \geq 2$ there is a finite set $\Gamma$ which contains elements $x_{1}, x_{2}, \ldots x_{k}, y$ among others, and such that any state $p$ on $\Gamma$ which assigns $k$ distinct values to $x_{1}, x_{2}, \ldots x_{k}$. assigns a different value to $y$. Also, taking the disjunction $\bigvee_{k=2}^{n} \mathcal{A}_{k}$, we obtain by the same method that for each $n \geq 2$ there is a finite set $\Gamma_{n}$ such that every non constant state $p$ on $\Gamma_{n}$ has at least $n$ values. We shall give below an explicit construction of $\Gamma_{n}$ in a somewhat more restricted context. ${ }^{1}$

So far we have used only the continuity of $p$, which is proved by G1-G4, but Gleason's theorem puts more severe restrictions on states than continuity. Conceptually, one of the important outcomes of Gleason's theorem are the uncertainty relations. Casting it in our language it says that any two non-orthogonal, non-opposite directions cannot both have extreme probability values (zero or one) unless they are both zero. To see the finite version consider the opposite statement:

F3 There is a state $p$ such that

$\exists x, y(0<(x, y)<1) \wedge((p(x)=p(y)=1) \vee(p(x)=1 \wedge p(y)=0) \vee(p(x)=$ $0 \wedge p(y)=1))$

Since F3 is false we can prove the following: Given any $x, y$ with $0<(x, y)<1$ there is a finite set $\Gamma$ such that $x, y \in \Gamma$, and every state $p$ on $\Gamma$ satisfies $p(x), p(y) \in$ $\{0,1\} \longleftrightarrow p(x)=p(y)=0$. This is the logical indeterminacy principle (Pitowsky 1998) which has been proved by an explicit construction, a simplified construction is given below. Note that this result is stronger than KS since it is constraining every probability distribution on $\Gamma$, and not merely the "truth values". It is "logical" in the sense that it follows from the orthogonality relations alone.

We can obtain more dramatic results of this kind, using the fact that by Gleason's theorem $p(x)=(x, W x)$. However, recall that this consequence is derived in the form $\forall \varepsilon>0 \forall x(|p(x)-(x, W x)|<\varepsilon)$. We should therefore be careful when moving to finite subsets. Let us begin with the simple example of a pure state. If we know that $p\left(z_{0}\right)=1$ then, by Gleason's theorem, $p(x)=\left|\left(z_{0}, x\right)\right|^{2}$ for all $x$. The statement $\left(p\left(z_{0}\right)=1\right) \wedge \exists x\left(p(x) \neq\left|\left(z_{0}, x\right)\right|^{2}\right)$ contradicts Gleason's theorem, but it is refuted

\footnotetext{
${ }^{1}$ This result has been used in Breuer (2002) to give an argument against the "way around" KS (Meyer 1999, Clifton and Kent 2000, see also, Pitowsky 1983,1985, and Appleby 2002).
} 
by showing that given $x$, and given $\varepsilon>0$ the condition $\left.|p(x)-|\left(z_{0}, x\right)\right|^{2} \mid<\varepsilon$ is satisfied. Hence, one cannot expect to be able to force the relation $p(x)=\left|\left(z_{0}, x\right)\right|^{2}$ for each $x$ on a finite set that contains it. Therefore, consider

$\mathbf{F} 4$ There is a state $p$ that satisfies $\left(p\left(z_{0}\right)=1\right) \wedge \exists x\left(\left.|p(x)-|\left(z_{0}, x\right)\right|^{2} \mid>\varepsilon\right)$.for some fixed $\varepsilon>0$.

The proposition F4 clearly contradicts Gleason's theorem. Using our method we conclude: For all $\varepsilon>0$ and $z_{0}, x \in \mathbb{S}^{2}$ there is a finite set of directions $\Gamma$ such that $z_{0} x \in \Gamma$, and every state $p$ on $\Gamma$ satisfies: $p\left(z_{0}\right)=\left.1 \rightarrow|p(x)-|\left(z_{0}, x\right)\right|^{2} \mid<\varepsilon$. Obviously, this is also true for any finite number of directions beside $x$. The general case, that of a mixture $W$, follows the same pattern. Here it is not enough to specify the value of $p$ at one point $z_{0}$. Rather, five points are needed since, in general, $W$ is a $3 \times 3$, self adjoint, non negative matrix with trace unity. Given these points $z_{1}, \ldots, z_{5}$, and the values $p\left(z_{i}\right)=\alpha_{i}$, we find for each $x$ and $\varepsilon>0$ a finite set on which the conditions $p\left(z_{i}\right)=\alpha_{i}$ imply $|p(x)-(x, W x)|<\varepsilon$. In order construct this set of directions explicitly one can painstakingly follow the steps of the constructive proof of Richman and Bridges (1999), and "lift" the vectors in the proof. An alternative to this tedious procedure is presented in the third section below, where there is a generic algorithmic way to calculate such graphs (and to demonstrate that Gleason's theorem, and theorems like it, have a constructive proof).

All these results are easily extendable to any real or complex Hilbert space of a finite dimension $n \geq 3$, and they are significant for the Bayesian approach developed in Pitowsky (2003). The results proved here imply that there are finite quantum gambles in which a rational agent is forced to bet in accordance with the numerical values of quantum probability, or very near them.

The inverse of these compactness results is the claim that there are very large subsets $\Omega \subset \mathbb{S}^{2}$ on which 0 -1 valued states exist. The "size" of such possible $\Omega$ depends on set-theoretic assumptions. For example, if the continuum hypothesis is assumed to hold, there is an $\Omega$ whose intersection with every major circle $C$ in $\mathbb{S}^{2}$ satisfies $|C \backslash \Omega \cap C| \leq \aleph_{0}$. Weaker assumptions lead to "smaller" sets (Pitowsky $1983,1985)$.

\section{Some COnstructions}

In this section we shall be using rays (one dimensional subspaces) rather than unit vectors and take states to be defined on them. Given a Hilbert space $\mathbb{H}$, the assumption $p(\alpha x)=p(x)$ for every scalar $\alpha,|\alpha|=1$ and every unit vector $x \in \mathbb{H}$ imply that $p$ actually depends on the ray and not on the unit vector we choose to represent it. Our first aim is to prove the "logical uncertainty principle". The proof here is simpler than Pitowsky (1998) and is based on the "lifting" of the vectors in an argument of Piron (1976).

Theorem 2. Let $a$ and $b$ be two non orthogonal rays in a Hilbert space $H$ of finite dimension $\geq 3$. Then there is a finite set of rays $\Gamma(a, b)$ such that $a, b \in \Gamma(a, b)$ and such that a state $p$ on $\Gamma(a, b)$ satisfy $p(a), p(b) \in\{0,1\}$ only if $p(a)=p(b)=0$.

Proof. First, consider the three dimensional real space $\mathbb{R}^{3}$. If $z$ and $q$ are two rays in that space there is a unique great circle which they determine. Let $q^{\prime}$ be the ray orthogonal to both $z$ and $q$ and let $q^{\prime \prime}$ be the ray orthogonal to both $q$ and $q^{\prime}$. Now, consider great circle through $q$ and $q^{\prime}$ ( figure 1 ). If $r$ is any ray through this great 
FiguRE 1.

FiguRE 2.

circle then $r \perp q^{\prime \prime}$. Let $l$ be the ray orthogonal to both $r$ and $q^{\prime \prime}$. The orthogonality relations between $z, q, q^{\prime}, q^{\prime \prime}, l, r$ is given in the graph $G=G(z, q, r)$ (figure 2). Subsequently we shall loosely identify sets of rays with their orthogonality graphs. If $p$ is a state defined on the rays in the graph $G$ then $p(z)=1$ entails $p(q) \geq p(r)$. Indeed, $p(q)+p\left(q^{\prime}\right)+p\left(q^{\prime \prime}\right)=p(r)+p(l)+p\left(q^{\prime \prime}\right)=1$. Also, since $p(z)=1$ we have $p\left(q^{\prime}\right)=0$. Hence $p(q)=p(r)+p(l) \geq p(r)$.

The relation between the points $z, q$, and $r$ can be best depicted on the projective plane, where $z$ is taken as the pole of projection (figure 3). In the projective plane great circles appear as straight lines, and latitudes (relative to $z$ as the pole) appear as concentric circles. In the projective plane $q$ is on a line through $z$, call this line $\mathcal{L}(z, q)$, and $r$ is on the line through $q$ which is perpendicular to $\mathcal{L}(z, q)$ at $q$.

Next, consider three points $z, q, r$ which do not necessarily have that relation. Assume only that $\measuredangle z q<\measuredangle z r$ so that if $z$ is the north pole, then $r$ is more to the south than $q$. In this case we can find a finite sequence of points $q_{1}, q_{2}, \ldots, q_{m}$ with 
FiguRE 3.

FIGURE 4.

$q_{1}=q$ and $q_{m}=r$ and such that $q_{k+1}$ is on the line perpendicular to $\mathcal{L}\left(z, q_{k}\right)$ for $k=1,2, \ldots, m-1$. A case with $m=5$ is considered in figure 4 . The number $m$ of intermediate points depends on the difference $\measuredangle z r-\measuredangle z q$ and on the respective longitude of $q$ and $r$.

Given this set of vectors we can construct for each $k$ a graph $G_{k}=G\left(z, q_{k}, q_{k+1}\right)$ in which $z, q_{k}, q_{k+1}$ play the role of $z, q, r$ respectively (note, $z$ is the same throughout). Let $G^{\prime}(z, q, r)=\bigcup_{k=1}^{m-1} G_{k}$ then any state on $G^{\prime}$ that satisfy $p(z)=1$ also satisfy

$$
p(q)=p\left(q_{1}\right) \geq p\left(q_{2}\right) \geq \ldots \geq p\left(q_{n}\right)=p(r)
$$

To finish the proof for $\mathbb{R}^{3}$ let $a, b$ be two non orthogonal rays. We can always choose a sequence of rays $c_{1}, c_{2}, \ldots, c_{n}$ such that $\measuredangle a b>\measuredangle b c_{1}>\measuredangle c_{1} c_{2}>\ldots>$ $\measuredangle c_{n-1} c_{n}$ and such that $a \perp c_{n}$. A case with $n=3$ is depicted in figure 5.Consider $b$ as a pole (projection point) and construct a graph $G_{0}^{\prime}=G^{\prime}\left(b, c_{1}, a\right)$ which is like $G^{\prime}(z, q, r)$ with $b, c_{1}, a$ play the role of $z, q, r$ respectively. If $p$ is a state on the rays 
FiguRE 5 .

in this graph with $p(b)=1$, then $p\left(c_{1}\right) \geq p(a)$. Consider $c_{1}$ as a pole and construct the graph $G_{1}^{\prime}=G^{\prime}\left(c_{1}, c_{2}, b\right)$, which is like $G^{\prime}(z, q, r)$, with $c_{1}, c_{2}, b$ play the role of $z, q, r$ respectively. For a probability function $p$ on $G_{1}^{\prime}$ which satisfy $p\left(c_{1}\right)=1$ we get $p\left(c_{2}\right) \geq p(b)$. Now construct the graph $G_{2}^{\prime}$ with $c_{2}$ as pole and $c_{3}, c_{1}$ play the role of $q, r$ respectively, and so on. Suppose that $p$ is a state on $G^{\prime \prime}(a, b)=\bigcup_{j=0}^{n-1} G_{j}^{\prime}$ the union of all these graphs. We shall show that if $p(a)=1$ then $p(b)<1$. Assume, by negation, that $p(a)=p(b)=1$ then, by construction $p\left(c_{1}\right) \geq p(a)$ so that $p\left(c_{1}\right)=1$. But then $p\left(c_{2}\right) \geq p(b)$ so that $p\left(c_{2}\right)=1$, and $p\left(c_{3}\right) \geq p\left(c_{1}\right)$ so that $p\left(c_{3}\right)=1$, and so on and we finally obtain $p\left(c_{n}\right)=1$. This is a contradiction since $a \perp c_{n}$. Hence $p(b)<1$.

Now, consider the ray $b^{\prime}$ which is orthogonal to $b$ in the plane spanned by $a$ and $b$, and let $b^{\prime \prime}$ be the ray orthogonal to both $b, b^{\prime}$. Repeat the construction of the graph with $b^{\prime}$ instead of $b$ to obtain the graph $G^{\prime \prime}\left(a, b^{\prime}\right)$, and add this to the previous graph. Let $p$ is a state on the graph $G^{\prime \prime}(a, b) \cup G^{\prime \prime}\left(a, b^{\prime}\right)$ with $p(a)=1$, then $f(b)<1$ and $f\left(b^{6}\right)<1$. But then we also have $p(b)>0$. Otherwise, $p(b)=0$ together with $p\left(b^{\prime \prime}\right)=0\left(\right.$ as $b^{\prime \prime} \perp a$ and $\left.p(a)=1\right)$ entail $p\left(b^{\prime}\right)=1$, contradiction. Hence $p(a)=1$ entails $0<p(b)<1$. Inverting the roles of $a$ and $b$ we construct a graph

$$
\Gamma(a, b)=G^{\prime \prime}(a, b) \cup G^{\prime \prime}\left(a, b^{\prime}\right) \cup G^{\prime \prime}(b, a) \cup G^{\prime \prime}\left(b, a^{\prime}\right)
$$

With $a^{\prime}$ a vector orthogonal to $a$ in the plane spanned by $a$ and $b$. Let $p$ be a state on $\Gamma(a, b)$. If $p(b)=1$ then $0<p(a)<1$, and if $p(a)=1$ then $0<p(b)<1$. Therefore $\Gamma(a, b)$ is the required set of rays in $\mathbb{R}^{3}$.

In the general case of a finite dimensional Hilbert space $\mathbb{H}$ we do the following: Given rays $a, b$ in $\mathbb{H}$ we consider them first as rays in a three dimensional subspace $\mathbb{H}^{\prime}$ of $\mathbb{H}$ and complete the construction there. Then we add to the finite set of rays in $\mathbb{H}^{\prime}$ additional $\operatorname{dim} \mathbb{H}-3$ orthogonal rays in the orthocomplement of $\mathbb{H}^{\prime}$. This completes the proof.

The the above construction entails that pure states should have strictly monotone behavior 
FiguRE 6.

Lemma 1. Given a ray $z$ in a Hilbert space $\mathbb{H}$ of a finite dimension $\geq 3$, and rays $a$ and $b$ such that $0<\measuredangle(a, z)<\measuredangle(b, z)$ then there is a finite set of rays $D(z, a, b)$, which contains $z, a$, and $b$, such that every state $p$ on $D(z, a, b)$ for which $p(z)=1$ also satisfies $p(a)>p(b)$.

Proof. Consider first $\mathbb{R}^{3}$. Given a ray $z$, let $q$ be any ray different from $z$ and not orthogonal to it. Consider once more the vectors in figute 1 and thier orthogonality graph $G=G(z, q, r)$ in figure 2 . Now denote

$$
D_{1}(z, q, r)=G(z, q, r) \cup \Gamma(z, l)
$$

where $\Gamma(z, l)$ is the set of rays in theorem 2 with $z, l$ fulfill the role of $a, b$. The situation is depicted in figure 6 . If $p$ is a probability function on $D_{1}(z, q, r)$ with $p(z)=1$ then, by construction, $p(q)=p(r)+p(l)$, and also $0<p(l)<1$, hence $p(q)>p(r)$. Since $\measuredangle(a, z)<\measuredangle(b, z)$ we can find a sequence $q_{0}, q_{1}, \ldots, q_{m}$ such that $q_{0}=a$ and $q_{m}=b$ and for all $k=1,2, \ldots m$ the ray $q_{k}$ is on the great circle through $q_{k-1}$ and $q_{k-1}^{\prime}$, the ray orthogonal to $z$ and $q_{k-1}$. Putting

$$
D(z, a, b)=\bigcup_{k=1}^{m} D_{1}\left(z, q_{k-1,} q_{k}\right)
$$

We get that if $p(z)=1$ then

$$
p(a)=p\left(q_{0}\right)>p\left(q_{1}\right)>\ldots>p\left(q_{m}\right)=p(b)
$$

If $\mathbb{H}$ is a Hilbert space $3 \leq \operatorname{dim} \mathbb{H}<\infty$, complete the construction first on the three dimensional space $\mathbb{H}_{1}$ spanned by $z, a$, and $b$ (in case they all lie in the same plane form $\mathbb{H}_{1}$ by adding any ray orthogonal to them). Subsequently add a set of orthogonal rays in $\mathbb{H}_{1}^{\perp}$ to complete the construction.

An immediate consequence of this theorem is the construction of a finite sets of rays on which states must take many values:

Corollary 1. Given a ray $z$ in a Hilbert space $\mathbb{H}$ of a finite dimension $\geq 3$, and an integer $k$, there is a finite set of rays $\Lambda_{k}(z)$ such that every state $p$ on $\Lambda_{k}(z)$ for which $p(z)=1$ has at least $k$ distinct values. 


\section{The Effectiveness of Gleason's Theorem}

Recently, there has been an interesting discussion on the question whether Gleason's theorem has a proof which is acceptable by the standards of constructive mathematics (Hellman 1993, Billinge 1997). The discussion culminated when Richman and Bridges (1999) gave a constructive formulation and proof of the theorem. Our aim is to give a (much shorter) proof of the conditional statement: If Gleason's theorem is true than it must have an effective proof.

The result follows from a generic sequence of approximations of states on finite sets. As will become clear subsequently the approximations in question are determined by an algorithm. Again, we shall consider the three dimensional case but the results generalize immediately. To simplify matters we shall work with general frame functions, not (positive normalized) states. This means that we replace G1 and $\mathbf{G} 3$ respectively by the axioms:

G'1 There is a real constant $\gamma$ such that $\forall x p(x) \geq \gamma$.

G'3 There is a real constant $\delta$ such that for each orthonormal triple $x, y, z \in \mathbb{S}^{2}$ : $p(x)+p(y)+p(z)=\delta$

Let $e_{1}=(1,0,0), e_{2}=(0,1,0)$ and $e_{3}=(0,0,1)$ be the standard basis in $\mathbb{R}^{3}$ (or $\left.\mathbb{C}^{3}\right)$ and $b_{i j}=\frac{1}{\sqrt{2}}\left(e_{i}+e_{j}\right), 1 \leq i<j \leq 3$.

Denote by $\mathcal{F}$ the set of all functions $p: \mathbb{S}^{2} \rightarrow \mathbb{R}$ that satisfy G'1, G2 and G'3 and let $\mathcal{F}_{0}=\left\{p \in \mathcal{F} ; p\left(e_{i}\right)=p\left(b_{i j}\right)=0, i=1,2,3,1 \leq i<j \leq 3\right\}$. Then

Lemma 2. The following statements are equivalent:

(a) If $p \in \mathcal{F}$ there is a self adjoint operator $W$ in $\mathbb{R}^{3}$ such that $p(x)=(x, W x)$.

(b) Every element $p \in \mathcal{F}_{0}$ vanishes identically.

Proof. If $p \in \mathcal{F}_{0}$ then by (a) we have $p(x)=(x, W x)$. But then $\left(e_{i}, W e_{i}\right)=0$ and $\left(b_{i j}, W b_{i j}\right)=0$. The latter equation implies that $\left(e_{i}, W e_{j}\right)=0$ for $1 \leq i<j \leq 3$. Hence $W=0$ and therefore $p=0$.

Conversely, assume (b) holds, let $p \in \mathcal{F}$ and let $W$ be the symmetric matrix which satisfies the equations $p\left(e_{i}\right)=\left(e_{i}, W e_{i}\right)$ and $p\left(b_{i j}\right)=\left(b_{i j}, W b_{i j}\right)$ for $i=1,2,3,1 \leq$ $i<j \leq 3$. Denote $p_{0}(x)=p(x)-(x, W x)$. Then $p_{0} \in \mathcal{F}$ and $p_{0}\left(e_{i}\right)=p_{0}\left(b_{i j}\right)=0$, and therefore $p_{0} \in \mathcal{F}_{0}$. By (b) $p_{0}=0$ and thus $p(x)=(x, W x)$.

By Gleason's theorem (a) is true, so we can take (b) as our formulation of this theorem. In the following we shall work with the first order formalization of the field of real numbers, the theory of real closed fields, denoted by $\mathbf{R}$, or the formalization of the field of complex numbers, the theory of algebraically closed fields (with zero characteristic), denoted by $\mathbf{C}$. In both theories there is an effective elimination of quantifiers. This means that there is a (known) algorithm which, given any well formed formula as input, produces as output an equivalent formula without the quantifiers $\forall, \exists$. Consequently the theories are decidable: there is an algorithmic method to prove every true proposition in them ${ }^{2}$. Also, we shall denote by $\mathbf{R}_{-}$the section of $\mathbf{R}$ without multiplication (including only the addition operation and the inequality relation).

As a result of the elimination of quantifiers every definable set (without parameters) in $\mathbf{R}$ is a finite Boolean combination of sets of $n$-tuples, each defined by a rational polynomial inequality (or equality). Thus, for example, $\mathbb{S}^{2}$ is the

\footnotetext{
${ }^{2}$ For details on these theories see Shoenfeld (1967). For recent results on quantifier elimination see Basu (1999)
} 
set $\left\{\left(\alpha_{1}, \alpha_{2}, \alpha_{3}\right) ; \alpha_{1}^{2}+\alpha_{2}^{2}+\alpha_{3}^{2}=1\right\}$ and therefore definable in $\mathbf{R}$. Similarly $\mathbb{S}_{+}^{2}$, "the northern hemisphere", which is like $\mathbb{S}^{2}$ with the additional condition $\left(\alpha_{3}>0\right) \vee\left(\alpha_{3}=0 \wedge \alpha_{1}>0\right) \vee\left(\alpha_{3}=0 \wedge \alpha_{1}=0 \wedge \alpha_{2}=1\right)$, is also definable in $\mathbf{R}$. Similar remarks apply to $\mathbf{C}$ and the unit sphere in the complex, finite dimensional Hilbert spaces.

We shall consider the general set-up where $X$ is some definable set of $n$-tuples, and $Y$ is a definable set of $m$-tuples from $X$. We let $\mathcal{F}_{0}$ be the set of real-valued functions $p$ on $X$, such that $\sum_{i=1}^{m} p\left(x_{i}\right)=0$ for all $y=\left(x_{1}, \ldots, x_{m}\right) \in Y$. Our first aim is to prove the following: If every bounded function of $\mathcal{F}_{0}$ vanishes identically in every model of $\mathbf{R}$ (or $\mathbf{C}$ ) then this fact admits an effective proof.

The connection with Gleason's theorem is as follows: We let $X=\mathbb{S}_{+}^{2}$, and $Y$ is the set of triples from $X$ given by $Y=B \cup\left\{\left(e_{i}, e_{i}, e_{i}\right) ; i=1,2,3\right\} \cup\left\{\left(b_{i j}, b_{i j}, b_{i j}\right) ; 1 \leq\right.$ $i<j \leq 3\}$. Here $B$ is the set of orthogonal triples from $\mathbb{S}_{+}^{2}$ and $e_{i}, b_{i j}$ as in lemma 2 . $B$ is clearly definable since the inner product of $x_{1}=\left(\alpha_{1}, \alpha_{2}, \alpha_{3}\right), x_{2}=\left(\beta_{1}, \beta_{2}, \beta_{3}\right)$ is a polynomial in the $\alpha_{i}$ 's and $\beta_{i}$ 's. Also, the $b_{i j}$ 's are given by a simple formula (for example, $b_{12}=(\alpha, \alpha, 0)$ with $\left.2 \alpha^{2}-1=0\right)$. In sum, $Y$ is definable. In this case $\mathcal{F}_{0}$ is the set of real-valued functions $p$ which satisfy $p\left(x_{1}\right)+p\left(x_{2}\right)+p\left(x_{3}\right)=0$ for all $\left(x_{1}, x_{2}, x_{3}\right) \in Y$. By lemma 2 the conclusion $p=0$ for all $p \in \mathcal{F}_{0}$ is equivalent to Gleason's theorem.

Now for a natural number $k>1$ let

$\mathcal{S}_{k}(n)=\left\{S \subset\{0, \ldots, n\}^{m}: \mathbf{R}_{-} \vdash\left(\forall \alpha_{0}\right) \ldots\left(\forall \alpha_{n}\right)\left(\bigwedge_{s \in S}\left(\sum_{i=1}^{m} \alpha_{s(i)}=0\right) \rightarrow \bigvee_{j=1}^{n}\left(k\left|\alpha_{0}\right| \leq\left|\alpha_{j}\right|\right)\right)\right\}$

Let $\mathbf{T}$ stand for either $\mathbf{R}$ or $\mathbf{C}$ then we have:

Lemma 3. Let $X$ be a definable set of $n$-tuples, $Y$ a definable set of $m$-tuples from $X$, and $\mathcal{F}_{0}$ the set of real-valued functions $p$ on $X$, such that $\sum_{i=1}^{m} p\left(x_{i}\right)=0$ for all $y=\left(x_{1}, \ldots, x_{m}\right) \in Y$. Then the following are equivalent

(1) Every bounded function in $\mathcal{F}_{0}$ vanishes in every model of $\mathbf{T}$.

(2) For some $n, \mathbf{T} \vdash\left(\forall x_{0}\right)\left(\exists x_{1}\right) \ldots\left(\exists x_{n}\right) \bigvee_{S \in \mathcal{S}_{k}(n)} \bigwedge_{s \in S}\left(y_{s} \in Y\right)$ where $y_{s}=$ $\left(x_{s(1)}, \ldots, x_{s(m)}\right)$.

Proof. Let $p \in \mathcal{F}_{0}$. If (2) holds for $n$, then there is $S \in \mathcal{S}_{k}(n)$ such that $\sum_{i=1}^{m} p\left(x_{s(i)}\right)=$ 0 for all $s \in S$. By the definition of $\mathcal{S}_{k}(n)$ we have $\left|p\left(x_{0}\right)\right| \leq \frac{1}{k}\left|p\left(x_{j}\right)\right|$ for some $1 \leq j \leq n$. But by (2) for each $x_{0} \in X$ we can find such $x_{j} \in X$. We conclude therefore that if $\beta>0$ bounds $p$, so does $(1 / k) \beta$. Thus repeating the argument we have proved $p=0$.

Conversely, assume that (2) fails. Note that the formulas

$$
\phi_{n}\left(x_{0}\right)=\left(\exists x_{1}\right) \ldots\left(\exists x_{n}\right) \bigvee_{S \in \mathcal{S}_{k}(n)} \bigwedge_{s \in S}\left(\left(x_{s(1)}, \ldots, x_{s(m)}\right) \in Y\right)
$$

define an increasing chain of sets $W_{k}(n)=\left\{a ; \phi_{n}(a)\right\}$, so their complements $\overline{W_{k}}(n)=$ $\left\{a ; \neg \phi_{n}(a)\right\}$ form a decreasing chain, and by assumption no element is empty. By the compactness theorem (or Gödel's completeness theorem), there exists a model $\mathcal{M}$ of $\mathbf{T}$ and $a_{0} \in|\mathcal{M}|$ such that $a_{0} \in \bar{W}_{k}(n)$ for all $n$.

We now show that some $p \in \mathcal{F}_{0}(\mathcal{M})$ is non zero. For each $a \in X(\mathcal{M})$, let $c_{a}$ be a new (and different) constant symbol; let $\overline{\mathbf{R}}$ be the theory consisting of the 
axioms of $\mathbf{R}_{-}$and for each $\left(a_{1}, \ldots, a_{m}\right) \in Y(\mathcal{M})$ the axiom $\sum_{i=1}^{m} c_{a_{i}}=0$, and for each $a \in X(\mathcal{M})$ the axiom $\left|c_{a}\right|<k\left|c_{a_{0}}\right|$. Then $\overline{\mathbf{R}}$ is consistent, so it also has a model. Hence, there exists a function $g$ on $X(\mathcal{M})$ into an ordered Abelian group $(B,<)$ with $\sum g\left(a_{i}\right)=0$ for all $\left(a_{1}, \ldots, a_{m}\right) \in Y(\mathcal{M})$ and with $|g(a)|<k\left|g\left(a_{0}\right)\right|$ for all $a \in X(\mathcal{M})$. In particular, $b_{0}:=\left|g\left(a_{0}\right)\right|>0$. Also, there exists a unique homomorphism $H: B \rightarrow \mathbf{R}$ with $H(z) \geq 0$ whenever $z \geq 0$, and $H\left(b_{0}\right)=1$. Now $p=H \circ g$ is a nonzero element of $\mathcal{F}_{0}(\mathcal{M})$, and therefore (1) fails.

This establishes the existence of a constructive proof for Gleason's theorem in complex Hilbert spaces. In this case, the condition "every bounded function in $\mathcal{F}_{0}$ vanishes" holds in every model of $\mathbf{C}$, iff it holds in $\mathbb{C}^{3}$. This means that condition (2) of the theorem states that Gleason's theorem is true iff $\forall x_{0} \phi_{n}\left(x_{0}\right)$ is provable in $\mathbf{C}$ for some $n$. But $\mathbf{C}$ has an effective decision procedure.

In the real case there is some complication because we do not assume Gleaason's theorem for every model, but only for the standard model; though a posteriori it will follow for every model. Therefore, we have to bridge the gap between "all models of $\mathbf{R}$ " and the standard model $\mathbb{R}$ for which Gleason's theorem is known to be true. From now on we specialize to the concrete $Y$ relevant to Gleason's theorem. More specifically, we shall take $X$ to be the real projective plane, using its identification with the northern hemisphere $\mathbb{S}_{+}^{2}$ of the previous section (Figure 3 ). With this identification (and with the point at infinity added) $X$ is compact and $Y$ is closed. It follows that the sets $W_{k}(n)$, being projections of closed sets, are also closed sets, and so the sets, $\bar{W}_{k}(n)$ are open; but all we need is their measurability with respect to the uniform measure on the sphere, and the measurability of their intersections with major circles with respect to the uniform measure on the circle. Since $W_{k}(n)$ are definable sets, the subsets of the circle that will be mentioned below are finite unions of segments (i.e. arcs) and their measure is elementary.

Theorem 3. The conditions below, referring to the model $\mathbb{R}$, are equivalent.

(1) Every bounded function in $\mathcal{F}_{0}$ vanishes.

(2) For some $n, \mathbf{T} \vdash\left(\forall x_{0}\right)\left(\exists x_{1}\right) \ldots\left(\exists x_{n}\right) \bigvee_{S \in \mathcal{S}_{2}(n)} \bigwedge_{s \in S}\left(y_{s} \in Y\right)$ where $y_{s}=$ $\left(x_{s(1)}, \ldots, x_{s(m)}\right)$. (that is, for some $n, \bar{W}_{2}(n)=\emptyset$ )

(3) For some $n$, for every great circle $C$, the set $C \cap W_{4}(n)$ has normalized measure $>1 / 2$ in $C$.

(4) $\cap_{n \geq 1} \bar{W}_{8}(n)=\emptyset$.

Condition (2), while phrased for $\mathbb{R}$, is a single elementary statement; thus if true it holds in every real closed field, and this fact admits an elementary proof. So in the special case of Gleason's theorem, we can replace "in every model of $\mathbf{R}$ " by "in R.".

Proof. We will use the following kind of Fubini principle: Let $C_{z}$ be the great circle orthogonal to the point $z$ on the sphere. For $C=C_{z}$ let $\mu_{C}$ be the normalized uniform measure on $C_{z}$; and say that a property $P$ holds "for a majority of points" if $\mu_{C}(\{x: P(x)\}) \geq 1 / 2$. Let $C$ be a fixed great circle and suppose that a majority of points $z \in C$ are such that for a majority of points $q \in C_{z}$, the property $P(q)$ holds. Then, assuming $\{x: P(x)\}$ is measurable on the sphere, it has an area $\geq A$, where $A$ is the area of the unit sphere above the 45 th northern latitude. The reason

\footnotetext{
${ }^{3}$ Knowing the theorem for $\mathbb{C}$ automaticaly imply it for every model, since $\mathbb{C}$ is universal for countable models of $\mathbf{C}$; however, $\mathbb{R}$ does not have that property.
} 
is that the region $\{x: P(x)\}$ takes up $\geq 1 / 4$ of the area of a Mercator map of the sphere; and the region above the 45 latitude has the greatest distortion under this map. Moving to the projective plane $X$, we can say that $\{x: P(x)\}$ takes an area $\geq 1 / 2$ of $X$, when we use the area measure induced on $X$ by the Mercator map.

Claim 1. If $z \in \bar{W}_{k}(2 n+2)$, then a majority of $x \in C_{z}$ lie in $\bar{W}_{2 k}(n)$.

Indeed, in the projective plane model, a point $x$ on a given circle $C=C_{z}$, has a unique point $\operatorname{ort}(x)$ orthogonal to it on $C_{z}$. Suppose the claim fails. So let $z \in \bar{W}_{k}(2 n+2)$ and assume that a set of points of $C_{z}$ of measure $>1 / 2$ lies in $W_{2 k}(n)$. Since the function ort is a measure-preserving bijection on $C_{z}$, the set $\left\{y: \operatorname{ort}(y) \in W_{2 k}(n)\right\}$ also has measure $>1 / 2$. So these two sets intersect; thus there exist $x, y \in C_{z}$ such that $x \perp y$ and $x, y \in W_{2 k}(n)$. But as $x, y, z$ form an orthogonal triple, $(x, y, z) \in Y$, so it is easy to see that $z \in W_{k}(2 n+2)$, a contradiction which proves the claim.

(4) implies (3): Assuming (4), there exists $n_{4}$ such that $W_{8}\left(n_{4}\right)$ has measure $>A$, that is, Mercator measure $>1 / 4$. Let $n_{3}=2 n_{4}+2$. Let $C$ be a great circle. Suppose, by negation, that a majority of points of $C$ lie in $\bar{W}_{4}\left(n_{3}\right)$. Then, by the claim, for each of these points $z \in \bar{W}_{4}\left(n_{3}\right) \cap C$, a majority of points $x$ of $C_{z}$ must lie in $\bar{W}_{8}\left(n_{4}\right)$. So this set has (Mercator) area $\geq 1 / 4$. Moving to the projective plane we get a contradiction. Thus (3) holds.

(3) implies (2): Assume (3) holds for $n=n_{3}$, and let $n_{2}=2 n_{3}+2$. If $y \in \bar{W}_{2}\left(n_{2}\right) \neq \phi$, let $C_{y}$ be the orthogonal great circle. Then by the claim a majority of points on $C_{y}$ lie in $\bar{W}_{4}\left(n_{3}\right)$, contradicting (3).

(2) implies (1): The same as in lemma 3.

(1) implies (4): As soon as there is $a_{0} \in \cap_{n \geq 1} \bar{W}_{8}(n)$, the proof of lemma 3 works and provides a non-vanishing bounded function in $\mathcal{F}_{0}$.

The theorem provides an effective procedures to prove $p=0$ in case every $p \in \mathcal{F}_{0}$ vanishes. The reason is that $\mathbf{R}$ (and $\mathbf{R}_{-}$) is decidable, which means that there is a computer program which proves evry true propositions of $\mathbf{R}$, in particular the proposition $\left(\forall x_{0}\right) \phi_{n}\left(x_{0}\right)$ for a suitable integer $n$. Any apparent ineffectiveness in the proof of theorem 3 is in some sense self-eliminating, as a posteriori one knows the existence of an effective proof too. Nevertheless, it may be worth remarking that all the sets occurring in the proof are definable in $\mathbf{R}$. These sets are known to have a simple structure, and there would be no difficulty in formalizing the proof in a very small part of Peano arithmetic.

As a consequence of theorem 1 we obtain:

Corollary 2. For any direction $x_{0}$ there is a finite, fixed size set $\Gamma \subset \mathbb{S}_{+}^{2}$ which include $x_{0}$, the $e_{i}$ 's and $b_{i j}$ 's such that any $p \in \mathcal{F}_{0}(-)$ satisfy $\left|p\left(x_{0}\right)\right| \leq \frac{1}{2}|p(x)|$ for some $x \in \Gamma$.

Here $\mathcal{F}_{0}(-)$ is the set of functions $p: \Gamma \rightarrow \mathbb{R}$ which satisfy the conditions $p\left(e_{i}\right)=p\left(b_{i j}\right)=0$ and $p(x)+p(y)+p(z)=0$ for every orthogonal triple $x, y, z \in \Gamma$. Iteration of this process yields a constructive proof of Gleason's theorem. It would be nice to give an explicit construction of the set $\Gamma$ with this property. As noted, there is a general algorithm to find it, but such algorithm may not be feasible to execute in practice since the decision procedure for $\mathbf{R}$ has a worst case doubly exponential time lower bound, see Basu (1999). 
We shall end with a few comments on the boundedness condition in Gleason's theorem. Suppose that we drop the requirement that the functions $p \in \mathcal{F}_{0}$ are bounded, then the conclusion $p=0$ is simply false. One can see this from the following cardinality consideration:

Denote by $\mathcal{G}$ the class of real functions $f$ satisfying $f(x)+f(y)+f(z)=0$ for every orthogonal triple $x, y, z \in \mathbb{S}_{+}^{2}$. By Gleason's theorem the cardinality of all bounded functions in $\mathcal{G}$ is $2^{\aleph_{0}}$. Note that $\mathcal{G}$ (including its unbounded functions) is closed under composition with any additive group homomorphism $\mathbb{R} \rightarrow \mathbb{R}$; i.e. if $g \in \mathcal{G}$ and $h: \mathbb{R} \rightarrow \mathbb{R}, h(x+y)=h(x)+h(y)$, then $h \circ g \in \mathcal{G}$. Let $p$ be a bounded element of $\mathcal{G}$ given by some non-zero matrix with trace zero. If one assumes the axiom of choice, there are $\beth_{2}$ additive group homomorphisms from $\mathbb{R}$ to $\mathbb{R}$. Composing them with $p$, we see that $\mathcal{G}$ is enormous, and so certainly Gleason's theorem fails without the boundedness requirement.

On the other hand consider a model of set theory in which the axiom of choice fails, Solovay's (1970) model, or if every set has the property of Baire say. Then every function $\mathbb{R} \rightarrow \mathbb{R}$ is continuous on a set whose complement is meager; if it is also a group homomorphism, it will be continuous everywhere. Thus, with this kind of negation of the axiom of choice, it is not implausible that the boundedness of frame functions might be automatic. Coming back to the remark at the end of section 1 , we also note that in such models there are no large $\Omega \subset \mathbb{S}^{2}$ on which 0-1 valued states exist (Shipman 1990).

It seems instructive at all events to compare Lemma 3 to what one obtains when $Y$ satisfies the stronger assumption, that every function (bounded or otherwise) vanishes. Then one can replace in Lemma $3(2)$ the reference to $S_{k}(n)$ by

$\mathcal{S}_{\infty}(n)=\left\{S \subset\{0, \ldots, n\}^{m}: \mathbf{R}_{-} \vdash\left(\forall \alpha_{0}\right) \ldots\left(\forall \alpha_{n}\right)\left(\bigwedge_{s \in S}\left(\sum_{i=1}^{m} \alpha_{s(i)}=0\right) \rightarrow\left(\alpha_{0}=0\right)\right)\right\}$

The proof is the same as that of Lemma 3 , mutatis mutandis, noting that when $B$ is a divisible Abelian group and $0 \neq b \in B$, there exists a homomorphism $H: B \rightarrow \mathbf{R}$ with $H(b) \neq 0$ (not necessarily order preserving.)

Acknowledgement 1. IP acknowledges the support of the Israel Science Foundation (grant 879/02).

\section{References}

Appleby, D.M. (2002) "Existential Contextuality and the Models of Meyer, Kent and Clifton" Phys. Rev. A 65, 022105.

Basu, S. (1999) New results on quantifier elimination over real closed fields and applications to constraint databases Journal of the ACM 46, 537-555.

Bell ,J. L. (1996) "Logical reflections on the Kochen-Specker Theorem" in Clifton, R. (ed) Perspective on Quantum Reality pp 227-235, Dordrecht, Kluwer.

Bell, J. S. (1966): "On the Problem of Hidden Variables in Quantum Mechanics", Reviews of Modern Physics 38, 447-452; reprinted in Bell, J S. (1987): Speakable and Unspeakable in Quantum Mechanics (Cambridge; Cambridge University Press)

Billinge, H. (1997) "A Constructive Formulation of Gleason's Theorem" Journal of Philosophical Logic 26, 661-670. 
Breuer, T. (2002) "Another No-Go Theorem for Hidden Variable Models of Inaccurate Spin Measurement" Philosophy of Science Association 18th Biennial Meeting - PSA 2002, http://philsci-archive.pitt.edu/view-200204.html.

Clifton, R. (1993) "Getting Contextual and Nonlocal Elements-of-Reality the Easy Way" American journal of Physics 61, 443-447.

Clifton, R. and Kent, A. (2000) "Simulating Quantum Mechanics by Non-Contextual Hidden Variables" Proceedings of the.Royal Society of London A 456, 2101-2114.

Cooke, R. Keane, M. and Moran, W. (1984) "An Elementay Proof of Gleason's Theorem" Mathematical Proceedings of the Cambridge Philosophical Sosiety 98, 117-128.

Fine, A. and Teller, P. (1978) Algebraic Constraints on Hidden Variables. Foundations of Physics 8 629-636.

Gleason, A. M. (1957) Measures on the Closed Subspaces of a Hilbert Space. Journal of Mathematics and Mechanics 6, 885-893.

Held, C. (2000) "The Kochen and Specker Theorem" Stanford Encyclopedia of Philosophy http://plato.stanford.edu/

Hellman, G. (1993) "Gleason's Theorem is Not Constructively Provable" Journal of Philosophical Logic 22, 193-203.

Kochen, S. and Specker, E. P. (1967) "The Problem of Hidden Variables in Quantum Mechanics". Journal of Mathematics and Mechanics 17, 59-87.

Meyer, D. A. (1999) "Finite Precision Measurement Nullifies the Kochen-Specker Theorem" Physical.Revew.Letters 83, 3751-3754.

Piron, C. (1976) Foundations of Quantum Physics, (Reading MA, AddisonWeseley).

Pitowsky, I. (1982) "Substitution and Truth in Quantum Logic", Philosophy of Science 49, 380-401.

Pitowsky, I. (1983) "Deterministic Model of Spin and Statistics", Physical Review D27, 2316-2326.

Pitowsky, I. (1985) "Quantum Mechanics and Value Definiteness", Philosophy of Science 52, 154-156.

Pitowsky, I. (1998). "Infinite and Finite Gleason's Theorems and the Logic of Indeterminacy", Journal of Mathematical Physics 39, 218 - 228.

Pitowsky, I. (2003) "Betting on the Outcomes of Measurements: A Bayesian Theory of Quantum Probability" Studies in the History and Philosophy of Modern Physics 34, 395-414.

Richman, F. and Bridges, D. (1999) "A Constructive Proof of Gleason's Theorem Journal of Functional Analysis 162, 287-312.

Shipman, J. (1990) "Cardinal Conditions for Strong Fubini Theorems" Transactions of the American Mathematical Society 321, 465-481.

Shoenfield, J. R. (1967) Mathematical Logic (Reading MA, Addison-Weseley).

Solovay, R. M. (1970) "A model of set-theory in which every.set of reals is Lebesgue measurable", Annals of Mathematics 92, 1-56.

Department of Mathematics, the Hebrew University

E-mail address: ehud@math.huji.ac.il

Department of Philosophy, The hebrew University

E-mail address: itamarp@vms.huji.ac.il

URL: http://edelstein.huji.ac.il/staff/pitowsky/ 


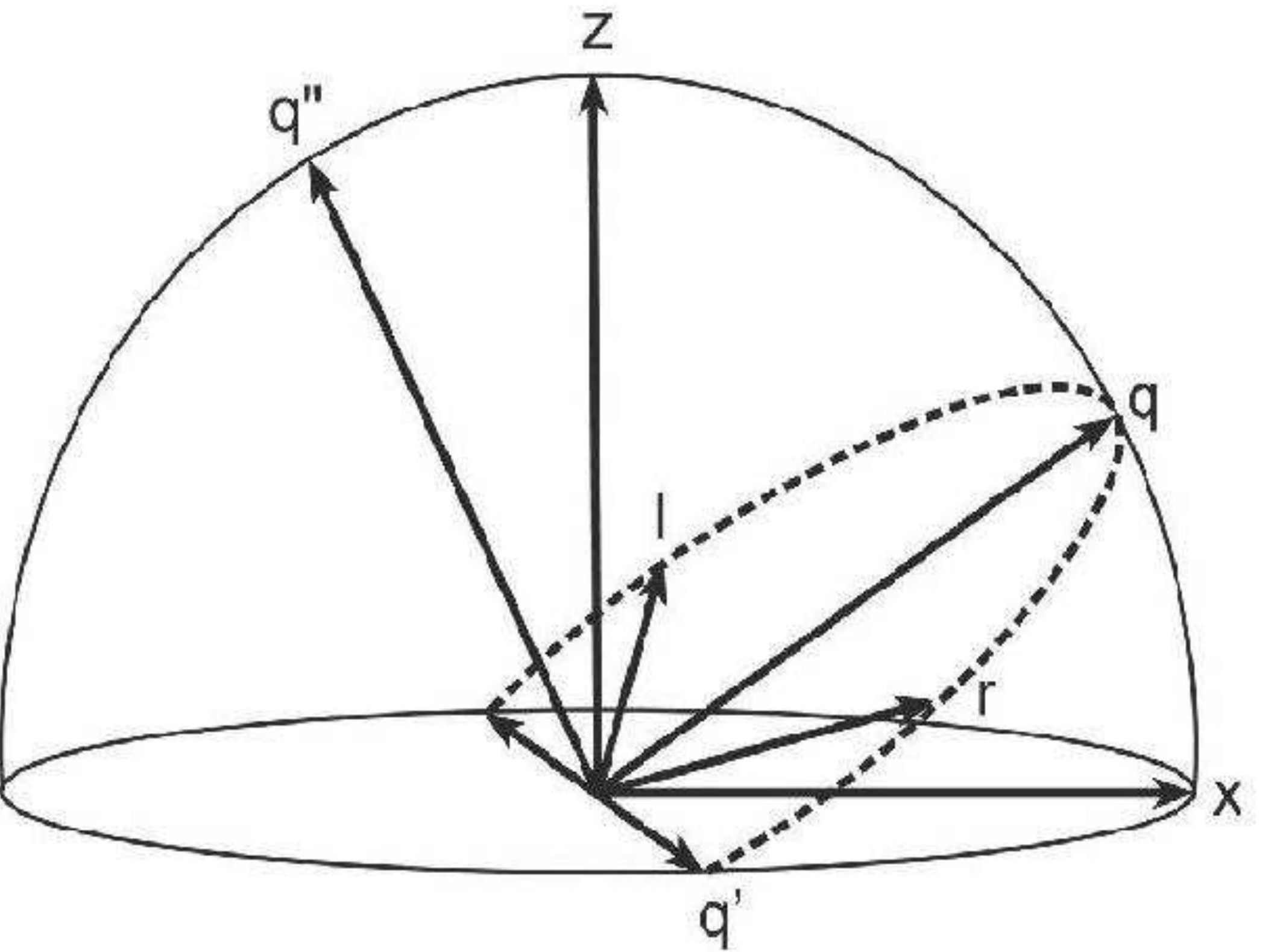




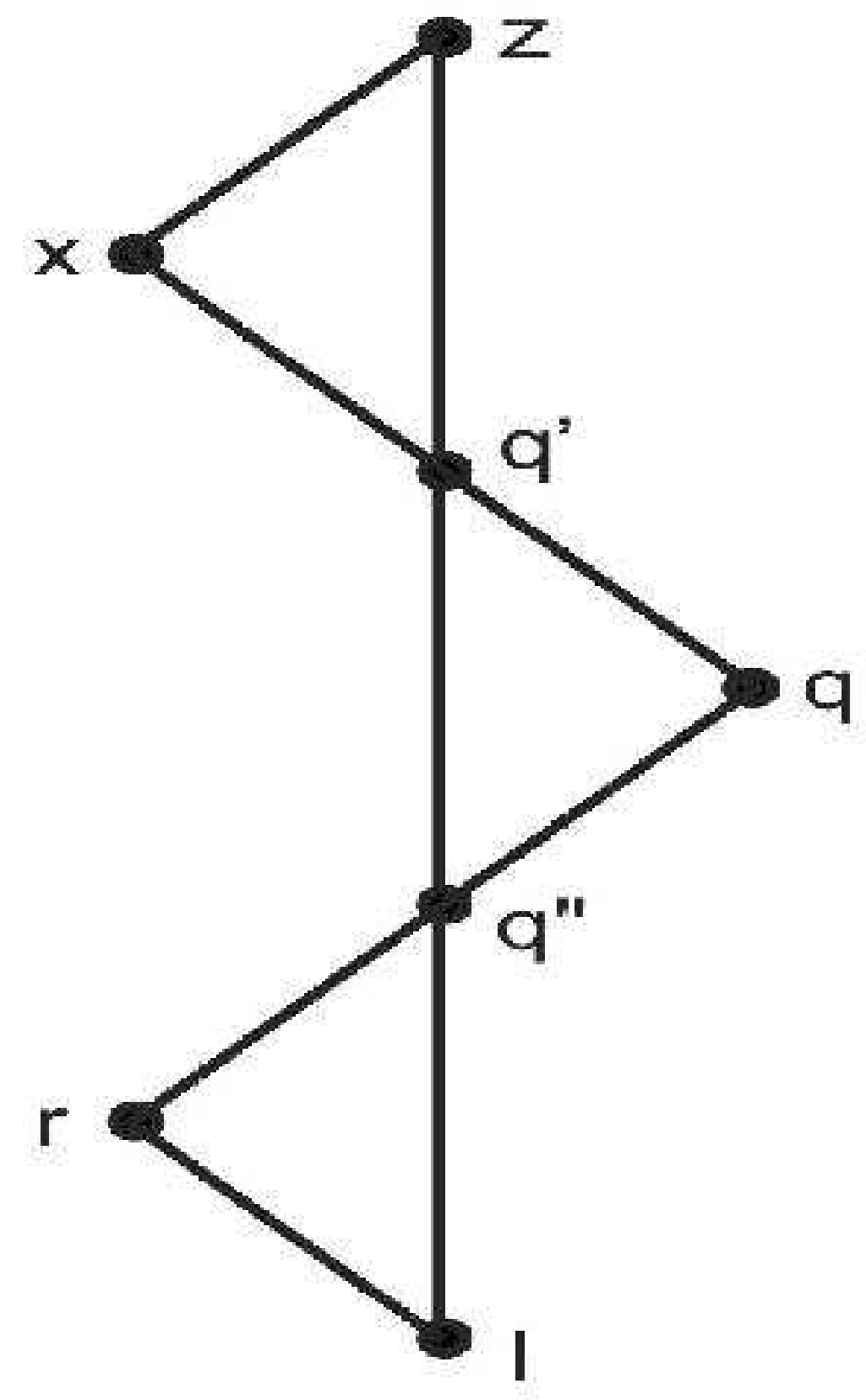




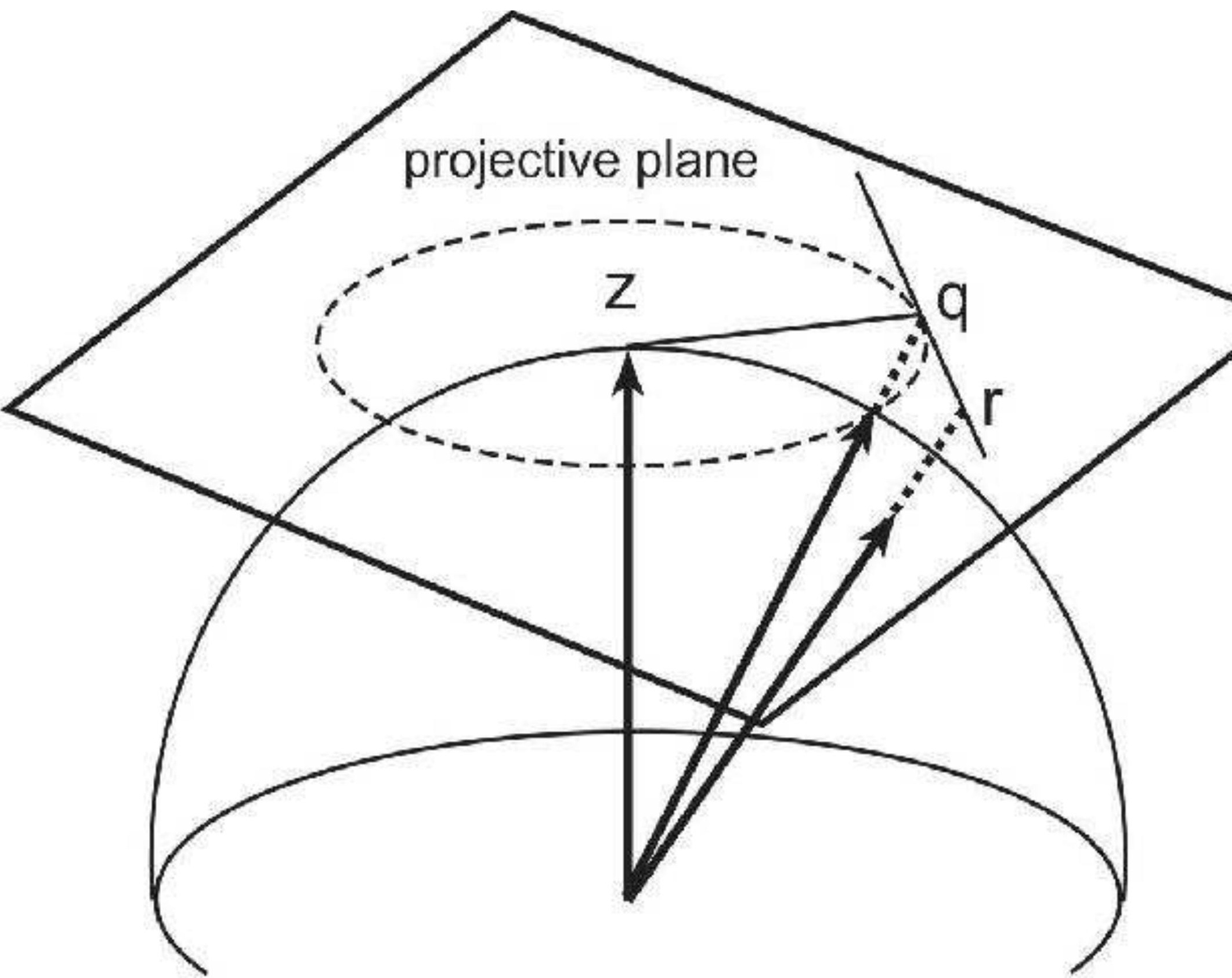




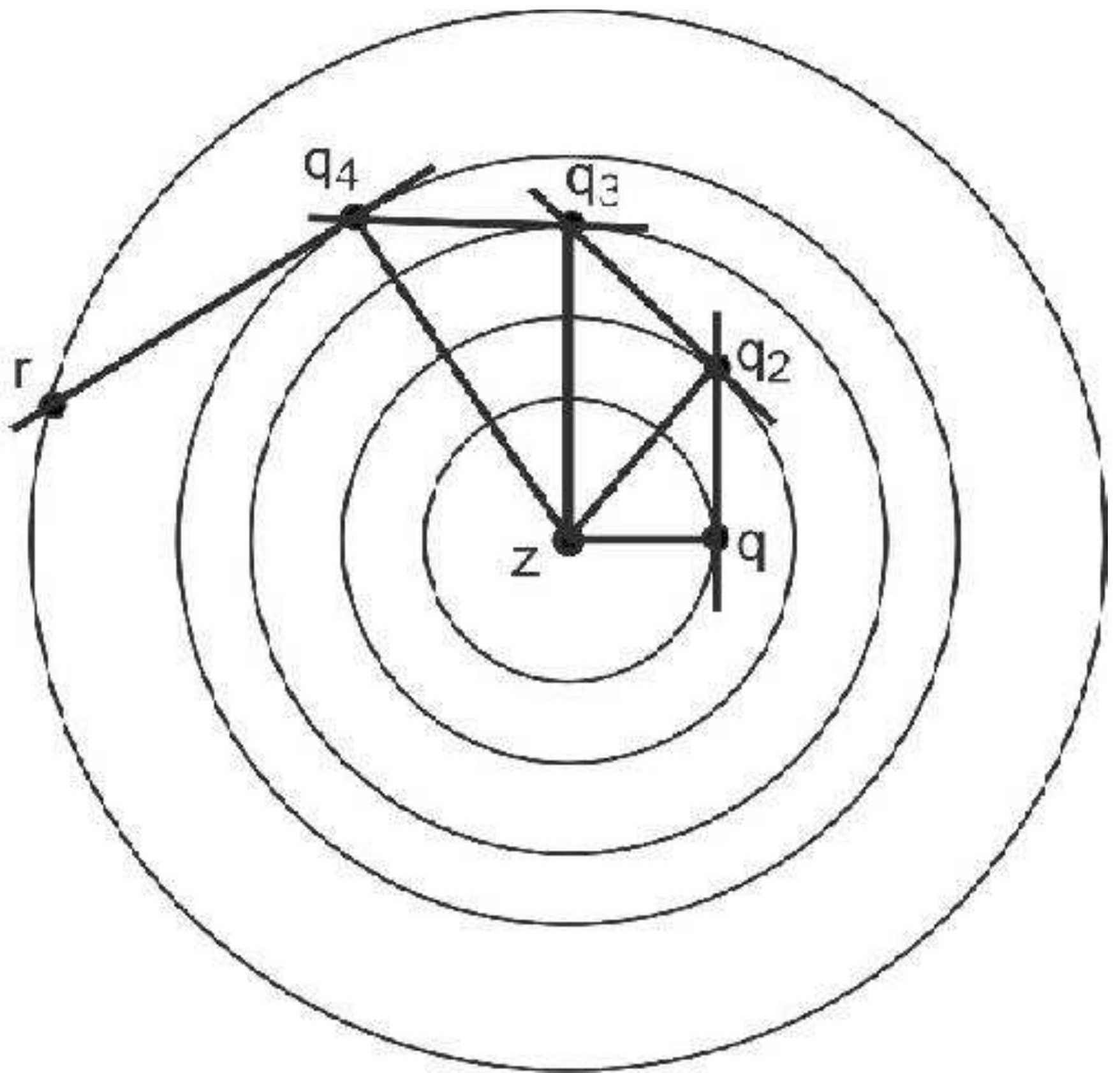




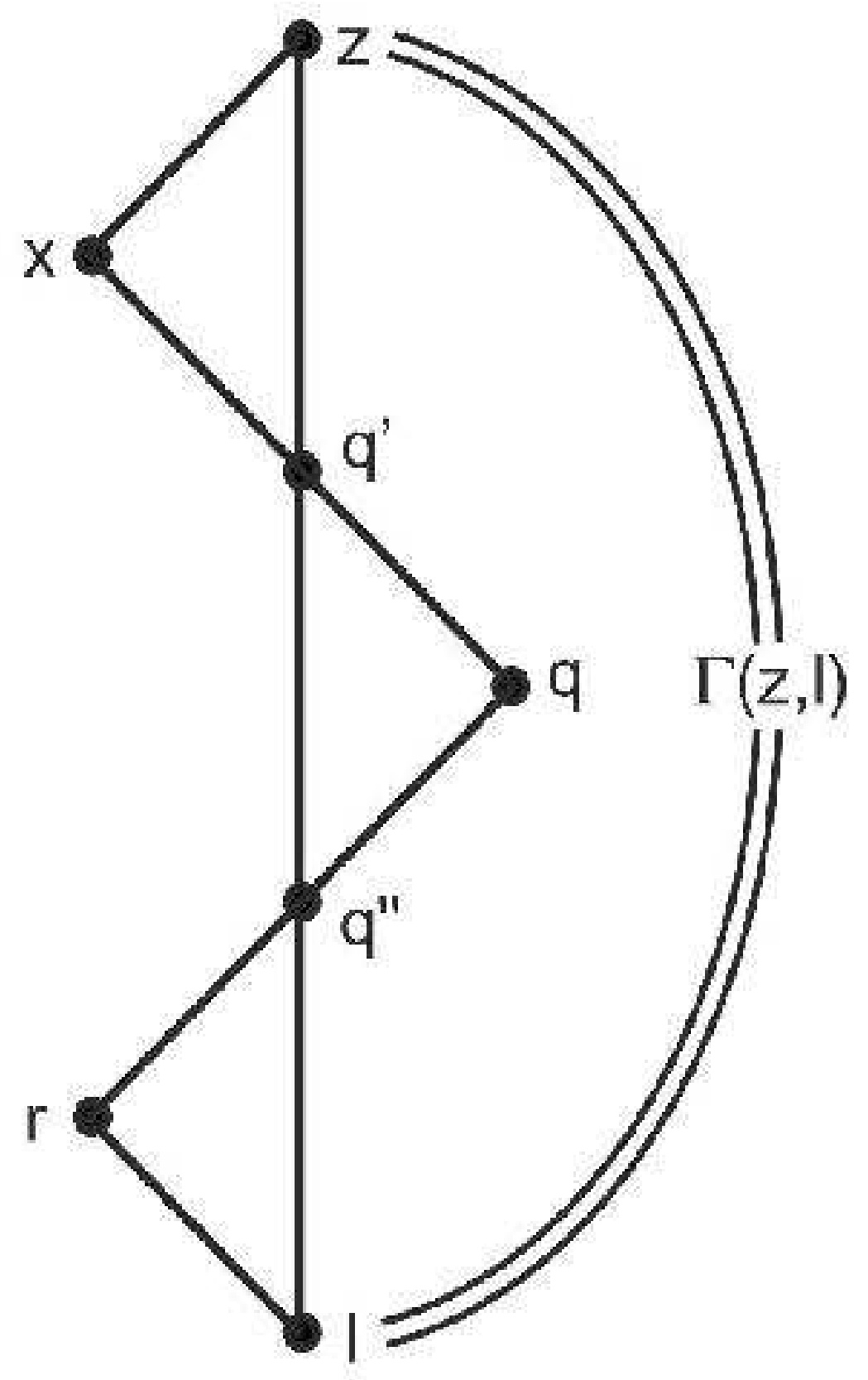

